\title{
On-Fiber 3D Printing of Photonic Crystal Fiber Tapers for Mode Field Diameter Conversion
}

\author{
Andrea Bertoncini, Vijayakumar P. Rajamanickam, Carlo Liberale \\ Biological and Environmental Science and Engineering (BESE), King Abdullah University of Science and Technology (KAUST), Thuwal \\ 23955-6900, Saudi Arabia
}

The large mismatch between the Mode Field Diameter (MFD) of conventional single-mode fibers (SMFs) and the MFD of highly nonlinear Photonic Crystal Fibers (PCFs), that can be down to $1.5 \mu \mathrm{m}$, or Large Mode Area PCF, that can be up to $25 \mu \mathrm{m}$, would require a substantial fiber mode size rescaling in order to allow an efficient direct coupling between PCFs and SMFs. Over the years different solutions have been proposed, as fiber splicing of SMF to PCF. However these procedures are not straightforward, as they involve developing special splicing recipes, and can affect PCF optical properties at the splice interface [1].

Here we propose on-fiber Two-Photon Lithography (TPL) [2,3] fabrication of polymer PCF-based waveguiding structures to create ultra-compact and fiber-integrated beam shaping devices. In particular, we apply this approach to the fabrication of a PCF taper for optical fiber MFD conversion [4]. Thanks to the versatility of TPL, 3D structures with arbitrary geometry can be fabricated, and by considering the reciprocity principle, tapers for adiabatic up-scale and down-scale mode size converters can be designed.
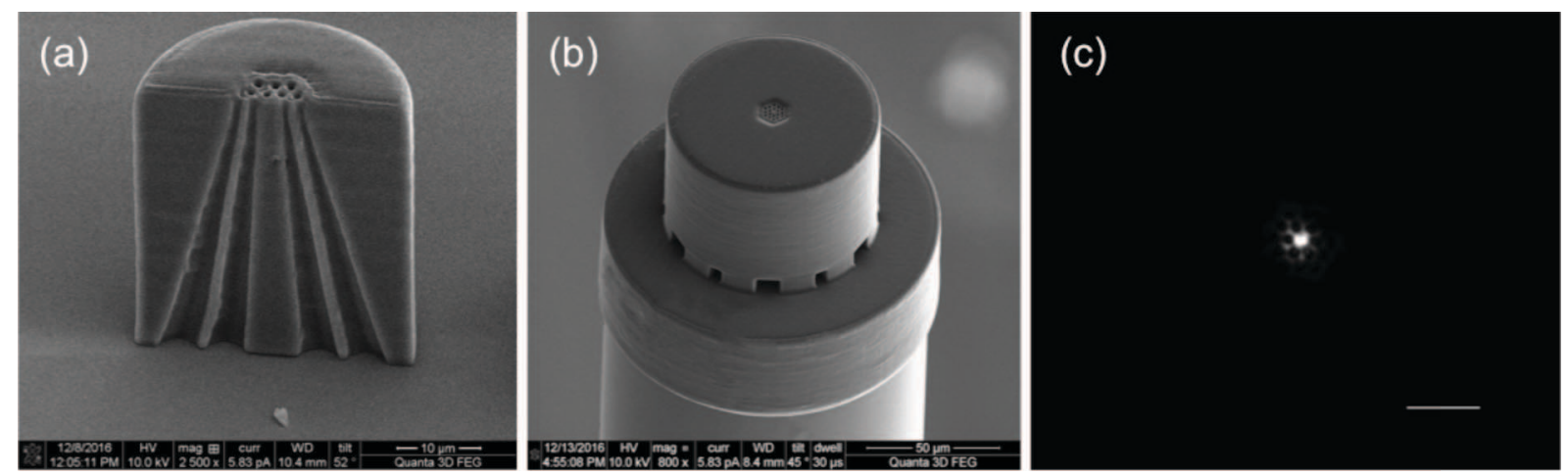

Fig. 1 (a) section of the PCF taper fabricated on to a glass slide to show the internal structure; (b) final PCF taper structure fabricated on top of the single-mode fiber; (c) measured output intensity distribution for the PCF taper Scale bar: $10 \mu \mathrm{m}$.

A down-taper has been designed to convert the fundamental mode of a SMF (1060XP, Thorlabs), with a $6.2 \mu \mathrm{m}$ MFD at $\lambda=1060 \mathrm{~nm}$, to a $2 \mu \mathrm{m}$ mode size, a typical value for highly nonlinear PCFs. In order to provide a good modal matching to this single-mode fiber, the initial pitch $\Lambda$ of the tapered PCF structure has been chosen to be $6.5 \mu \mathrm{m}$. The fill factor has been kept constant at $d / \Lambda=0.4$ along the taper, in order to maintain single-mode regime for the PCF [5]. A taper angle of $0.045 \mathrm{rad}$ has been chosen for the structure, in order to satisfy the adiabaticity criterion for waveguide tapers [6]. With these parameters the resulting PCF taper length is $50 \mu \mathrm{m}$.

Fabrication of the PCF taper (Fig. 1a-b) has been performed with a commercial TPL system (Nanoscribe $\mathrm{GmbH}$ ). Fabrication time for this taper was about 10 minutes. The polymer PCF taper structure has a very good adhesion to the glass optical fiber and is sturdy enough to withstand touching hard surfaces with the tip of the fabricated fiber.

The PCF taper output mode has been characterized by imaging on to a CCD camera (Fig. 1c). The optical intensity distribution has a FWHM of $2 \mu \mathrm{m}$ and is very well localized at the core of the final PCF section, demonstrating efficient downscaling of the original single mode fiber fundamental mode.

\section{References}

[1] NKT Photonics Application Note V1.0 November 2009, "Splicing Single Mode PCFs", http://www.nktphotonics.com/

[2] C. Liberale et al., "Micro-optics fabrication on top of optical fibers using two-photon lithography," IEEE Photon. Technol. Lett. 22, 474

(2010).

[3] S. Bianchi et al. "Focusing and imaging with increased numerical apertures through multimode fibers with micro-fabricated optics," Opt. Lett. 38, 4935-4938 (2013).

[4] G. E. Town and J. T. Lizier, "Tapered holey fibers for spot-size and numerical-aperture conversion,” Opt. Lett. 26, 1042-1044 (2001).

[5] P. Russell, "Photonic Crystal Fibers," Science 299, 358-362 (2003).

[6] J. D. Love, Electron. Lett. 23, 993 (1987). 